\title{
Countability and Self-Identity
}

\author{
Adrian Heathcote
}

30th September 2021

\begin{abstract}
The Received View of particles in quantum mechanics is that they are indistinguishable entities within their kinds and that, as a consequence, they are not individuals in the metaphysical sense and self-identity does not meaningfully apply to them. Nevertheless cardinality does apply, in that one can have $n>1$ such particles. A number of authors have recently argued that this cluster of claims is internally contradictory: roughly, that having more than one such particle requires that the concepts of distinctness and identity must apply after all. A common thread here is that the notion of identity is too fundamental to forego in any metaphysical account. I argue that this argument fails. I then argue that the failure of individuality and identity applies also to macroscopic physical objects, that the problems cannot be constrained to apply only within the microscopic realm.
\end{abstract}

In the mid-1920s it was discovered that statistical ensembles of bosons and fermions obey a different statistics than that which Boltzmann had found for classical ensembles, with the implication that these bosons and fermions were indistinguishable entities within their kinds. This was then fine-tuned by the discovery of the Pauli exclusion principle in 1925. Pauli's original formulation of the principle was then supplanted in 1926 by Heisenberg and Dirac, independently, with the statement that the state space for bosons was a symmetric tensor space and that of fermions was an antisymmetric space. From this the Pauli exclusion principle follows (Dirac (1926)). ${ }^{1}$ Despite this one can have a number of such particles, fermions and bosons - for example two, three, or $n$, indistinguishable electrons, or $n$ indistinguishable photons. This is often called the Received View of quantum

\footnotetext{
${ }^{1}$ For fermions this means that the many-particle wave function is antisymmetric in the exchange of the spin-spatial coordinates for any pair of particles. 'An antisymmetrical eigenfunction vanishes identically when two of the electrons are in the same orbit. This means that in the solution of the problem with antisymmetrical eigenfunctions there can be no stationary states with two or more electrons in the same orbit, which is just Pauli's exclusion principle.' Dirac (1926) 669-70.
} 
mechanics (henceforth QM), or, within physics, the orthodox view. ${ }^{2}$ But if one can have indistinguishable particles that are not numerically the same, then the Leibniz principle of the identity of indiscernibles (henceforth PII) cannot apply to them and, it is argued, the particles are not 'individuals'. 'Self-identity' seems then also to be in doubt, for if PII is not available to guarantee self-identity, by sameness of properties, then what can guarantee it? (Haecceities can't guarantee it unless we can independently guarantee that haecceities exist. ${ }^{3}$ )

We can summarise the main aspects of the Received View in the following claims:

1. Particles within their kinds are indistinguishable entities.

2. One can have $n$ such particles, i.e. a collection of them can have a cardinality.

3. They cannot be enumerated, i.e. paired with ordinals, since that would contradict 1 . For the same reason they cannot be given distinct labels. This can be put in general terms: there can be no bijection from any sequence of $n$ ordinals to $n$ indistinguishable particles.

From the Received View the claim that they are not individuals and do not have self-identity is claimed to follow.

The Received View was first articulated by Hermann Weyl in his 1930 work Gruppentheorie und Quantenmechanik, translated as Weyl (1931), with additional ideas presented in Weyl (1949). In particular it was Weyl who first showed how to fully decompose a tensor space into 'representation spaces' such as the symmetric and antisymmetric spaces (see Heathcote (2020)). The next most important contribution was made by Schrödinger in his (1950), (1952). Since then the Received View has been defended and discussed by many: for example ParkerRhodes (1981), Toraldo di Francia (1975), Dalla Chiara (1985), van Fraassen (1991), Dieks (2020), and many others. ${ }^{4}$ But central to any such discussion must be that of Stephen French and Décio Krause, in particular French and Krause (2006) and (2010). ${ }^{5}$ The view of particle indistinguishability in the technical part

\footnotetext{
${ }^{2}$ It has to be stressed that this aspect of QM is independent of interpretational issues. It was not deduced from ideas about 'superposition', or entanglement, which came later, contra a remark in Lowe (1998).

${ }^{3}$ If it is to be a property of a quantum system what Hermitean operator is there to measure it? What are its eigenstates?

${ }^{4}$ See also Dieks and Versteegh (2008), Dalla Chiara and Toraldo di Francia (1995) and Heathcote (2020).

${ }^{5}$ Strictly speaking French and Krause hold to an underdetermination thesis in (2006), but subsequent publications of Krause suggest a firmer adherence to the Received View. I don't follow them in this underdetermination thesis. My defence here concerns the Received View.
} 
of French and Krause (2006) was mostly focussed on finding a set of alternate mathematical tools for understanding indistinguishable entities, particularly the use of multisets (see Blizzard (1989)), an idea which had been sketched by Weyl as a notion of aggregates (for bosons) and monomial aggregates (for fermions) in his (1949). ${ }^{6}$ (Krause has adapted these to his purpose and has called the resulting entities quasi-sets.) However, as the title of their book makes clear their overall focus is on the idea of identity in QM. Since indistinguishability renders Leibniz's PII inapplicable there is no property that these particles have that can serve as a basis for any self-identity. In contrast, for French and Krause the situation for classical individuals - tables, chairs, people — was very different: for these things distinguishability, Leibniz's PII, and thus self-identity were taken to hold. They say:

. . . the existence of the individual and the establishment of self-identity are conceptually on a par in that we cannot envisage the possibility of one without the other. An individual is thus conceptually tied to its identity with itself in a manner in which it is not with other relations (and it is for this reason, perhaps, that identity is dismissed as unproblematic). (French and Krause (2006) p.14)

Their account of the Received View therefore sets up a two-sorted ontology of objects with a sharp difference between quantum non-individuals and macroscopic objects, with only the latter treated in line with orthodox metaphysics.

Here two problems loom for the Received View. One goes back to the three characteristics listed earlier: surely, someone might say — and several philosophers have said - the Received View involves a contradiction, for if one can have $n$ such particles then surely they must be different from one another and also not different from themselves, and thus identical with themselves. This argument has been made by Dorato and Morganti (2013) and Berto (2017). If this argument is right then the Received View collapses.

The second argument also focusses on the issue of self-identity: identity, it is claimed, is a fundamental concept and it must apply to everything, i.e. it must be universal. Its fundamentality is seen in that it cannot be defined in any terms that don't involve itself. Its universality is held to be contained in the very notion of an entity. Moreover self-identity requires the unity of the object, in some sense, and where is unity better exemplified than in elementary particles, which are by definition indivisible? As a corollary we might ask: how does it happen that macroscopic

\footnotetext{
${ }^{6}$ Such monomials had appeared earlier in Weyl (1931) ch IV. These monomials are such that the exponents are integers mod 2, i.e. occupation numbers are either 1 or 0 . It is in this sense that, as Weyl says, they encapsulate the Pauli exclusion principle. See Weyl (1949) p. 247.
} 
objects that are both individuals and have self-identity, are composed entirely of particles that are not individuals, according to French and Krause, and that lack self-identity? Surely if the macroscopic objects are individuals then so should be what constitutes them; or conversely, if each and every constituent particle is not an individual and lacks self-identity then so should the whole. Using a combination of these claims one can form an argument to the effect that elementary particles must be individuals and must have self-identity.

Thus there are now a number of papers that push back against the Received View, in particular Dorato and Morganti (2013), Jantzen (2011) (2017), Bueno (2014), and Berto (2017). A unifying theme of these criticisms is the importance of the concept of identity. As Otávio Bueno puts it: 'Identity is arguably one of the most fundamental concepts in metaphysics.' And concludes ' . . . it is very difficult to see how one can avoid it' (Bueno (2014) p. 41). And this is because identity is an essential condition of individuality. Identity it is said, is required to characterise an individual: nothing can be an individual unless it has well-specified identity conditions (see Lowe (1998) ch. 3 for an extended defence of this view). Here I will be mostly, but not exclusively, concerned with arguments in the last of these papers, Berto (2017), which has so far not received attention. ${ }^{7}$

A general worry with these criticisms is that, to a large extent, they are plainly directed against a theory in which elementary particles are assumed to be individuals - as on the Received View they are not. One suspicion must be that these authors are unconvinced by the arguments for the Received View and think that a little reminder of the 'importance of identity' will be enough to restore us to a healthy metaphysics. But if this is so for a good numbers of authors then it suggests that something has gone wrong in the articulation and defence of the Received View to leave so many unconvinced. I attempt to rectify this problem in $\S 3$. A second purpose here is to extend aspects of the Received View to macroscopic objects, using additional features of quantum mechanics. Thus, I claim, people, tables, chairs, planets, are not individuals and also lack self-identity. This was once an accepted metaphysical position (for example it was held by F. H. Bradley in his (1916)), but has not been heard much lately. We might call this the extended Received View. For those who are already alarmed by the Received View this will be an unwelcome turn, but I hope that it will come to be seen as a solution to a great many problems about identity that have long looked hopeless. At the very least a gauntlet is thereby thrown down.

\footnotetext{
${ }^{7}$ Much of my argument will also apply to that in Dorati and Morganti (2013). There is a comprehensive response to Bueno (2014) in Krause and Arenhart (2018b). There is a different line of argument against the Received View in the work of Simon Saunders and Fred Muller, either separately or together, but I've discussed their view elsewhere, as have many others, and so will only briefly touch on it here.
} 
To avoid confusion, I should note that I am not arguing for a closely related position, namely that the binary concept of Identity is fully eliminable from our metaphysics. This has been argued recently in Wehmeier (2012) (2014) with a reply by Trueman (2014). This is not the position I am seeking to defend. ${ }^{8} \mathrm{I}$ am happy to agree that a two-place predicate is definable in model theory and that it can be given the properties that are characteristic of our notion of identity. I am also happy to agree that identity claims are rife in mathematics and mathematical physics and even in that part of QM that pertains to particle indistinguishability. But acknowledging this does not entail that everything is an individual and has self-identity, and it is the latter that I deny.

I start by examining the claim that the concept of identity is both fundamental and universal. Then I move onto the idea that there must be a contradiction between the claim of the indistinguishability of elementary particles and the assertion that one can nevertheless have some number of them. In the course of this it is necessary to confront the idea that problems with referring to elementary particles can be overcome using the idea of arbitrary reference contained in Breckenridge and Magidor (2012). I then set out the reasons for extending the Received View to macroscopic objects.

\section{UNIVERSALITY AND CARDinality}

Let us consider the claim of the universality of self-identity, a view to which many philosophers appear to subscribe. One can get no more trenchant statement of the view than the following from Colin McGinn. 'What I want to suggest now is that identity has a universality and basicness that is hard to overstate; concepts don't get more basic than this - or more indispensable. Every object (or any other entity - property, function, you name it) is self-identical; identity is not a relation an entity can fail to stand in to something.' McGinn (2000) p. 9-10. And then also 'The law of identity is banality itself: "everything is identical to itself", or "for all $x, x=x$ "' Many of those who oppose the notion of indistinguishability share this idea. For example: 'Whatever $a$ is, " $a=a$ " has been taken as a trivial truth if there is any' (Berto (2017) p. 1). And on the question as to what a thing is, he quotes van Inwagen: 'An object is anything that can be the value of a variable, that is, anything we can talk about using pronouns, that is, anything (Van Inwagen 2002, 180)'.

We have then the claim that self-identity applies to everything — thus, presumably, waves, elementary particles, sets, and quantities. But a problem immediately

\footnotetext{
8 There are additional reasons why this dispute is tangential to my present concerns. Wehmeier treats self-identity as unproblematic - his focus is on the eliminability of the binary relation of identity.
} 
suggests itself: the extension of the self-identity predicate would then be a set that includes all things, and since there is no restriction placed on what a thing is, it would have to be a set that contains all sets. So the predicate of self-identity would have as its extension a universal set, a set of all sets. This is impossible.

A way around this problem, called plural quantification, has been described by George Boolos (Boolos (1984) (1985a) (1985b)), Richard Cartwright (Cartwright (1994) (2001)), and Timothy Williamson (Williamson (2003)). ${ }^{9}$ On this view quantifiers can range over everything, without giving rise to paradox. What is required is that the predicate 'non-self-membered' not have an extension that is a set. As Cartwright put it, also reminding us that this was Russell's own first reaction to his paradox :

There is no set that has as members all and only those things that are not members of themselves. But the things that are not members of themselves can simultaneously be the values of the variables of a firstorder language ... (Cartwright (1994) p. 3)

and Boolos:

We cannot always pass from a predicate to an extension of a predicate, a set of things satisfying a predicate. We can, however, always pass to the things satisfying the predicate (if there is at least one), and therefore we cannot always pass from the things to a set of them. (Boolos (1985b))

This is a view that appears to be held by Berto as well for he says "So understood, terms like "object" or "thing" stand for blanket notions: they provide no restriction to our quantifiers. Every $x$ is such that, if $x$ is a thing, then $x$ is $F$ " means nothing more and nothing less than "Every $x$ is $F$ ".' (Berto (2017) p. 4)

Let us then allow that we can have our quantifiers range over everything; can we say of everything that it is self-identical? Well we can take the previous quotes to be the answer: the predicate 'is self-identical' cannot determine a set of selfidentical things, though there may be things satisfying the predicate. On the pluralist view of quantification we are already saying this of 'is non-self-membered' and 'is quantifiable over'. However, once we break the connection between a plurality and that plurality as determining the membership of a set we also break the

\footnotetext{
9 A large literature has grown up on plural quantification, as can be seen from the collection Rayo and Uzquiano (2006) and the references given therein. An early criticism of the idea is Linnebo (2003). The more recent Linnebo and Rayo (2012) gives a guide to further issues. The Stanford Encyclopaedia entry on Plural Quantification was updated in 2017. My thanks to a reader of the journal for suggesting the inclusion of this issue.
} 
connection between the plurality and cardinality as a bijective function on that set to the cardinal numbers.

But the connection between cardinality and self-identity (or individuation) is precisely what is at issue in the arguments of Berto (2017) and Dorato and Morganti (2013) against the Received View. These authors hold that if we have a plurality of entities that are countable (i.e. have a cardinality $>1$ ) then they must be different from one another, i.e. non-identical. Here is the argument as expressed in Berto (2017):

That a sentence of the form " $a=b$ " is true, under this reading of "=", means that we need to count one thing: the thing named " $a$ ", which happens to be the thing named " $b$ " (flag again the naming issue, to be addressed below). That we, instead, count two things, means that that sentence is false. But then its negation, " $\neg(a=b)$ ", is true. So $a$ and $b$ are different. And if the concept of difference meaningfully applies to $a$ and $b$, the one of identity does as well. " $a=b$ " is meaningful together with its negation: adding or removing a negation in front of such a meaningful sentence cannot turn it into a meaningless one. The concept of identity cannot but apply to whatever the concept of difference applies to: if - to use Ryle's jargon - we have no category mistake in the latter case, we have no such mistake in the former. (Berto (2017) p. 10)

Clearly most of the work here is done in the first sentence, and in most of the paper this is what I will be focussing on, in particular the use of names in the argument.

We can find a similar argument in Dorato and Morganti, but without using names, instead relying on Saunders' use of Quine's weak discernibility relation. They say: 'Quantum particles can and should be regarded as primitively individuated, simply because they are countable at the level of the formalism (a fact used in the extant proofs of their weak discernibility) ... ' (Dorato and Morganti (2013) p. 606). So, in short, countability implies primitive individuation, via weak discernibility. ${ }^{10}$

Here I will put an argument to show that weak discernibility fails when one attempts to apply it to quantum particles, fermions or bosons. ${ }^{11}$ Here is the statement of weak discernibility:

\footnotetext{
10 A referee has argued that Dorato and Morganti's 'can and should' should be taken in a pragmatic sense. Since I confess I don't know how to do this consistent with Weyl's argument I leave it to the judgement of the reader.

11 I've given this argument elsewhere (Heathcote 2021) and applied it also to Black's spheres. Here I focus on its application to quantum particles.
} 
W-D Two objects are weakly discernible in $\mathscr{L}$ if they satisfy an irreflexive $\mathscr{L}$ formula in two free variables. (Saunders (2006) p. 57)

The problem comes when we look at how satisfaction is intended to work in this case. Since this comes from Quine we may use the most recent of Quine's definitions of satisfaction, in Quine (1986). Open sentences are satisfied by infinite sequences $s$ of objects drawn from the domain. ${ }^{12}$ Since weak discernibility is a dyadic relation we only need be concerned with ordered pairs: the sequence can be continued indefinitely just by repeating the second item in the sequence, as recommended by Quine (Quine (1986) p. 38). His favoured way of constructing such sequences from sets is to take singleton sets of objects from the domain, paired with ordinals. Thus ' $F x y$ ' is satisfied by pairs $\langle x, y\rangle$ which are the sets $\{\{\{x\}, 1\},\{\{y\}, 2\}\}$ (Quine (1986) 36 ff). Thus, again following Quine, if the predicate ' $F$ ' is to be understood as conquered, the open sentence ' $F x y$ ' is satisfied by the ordered pair $<$ Caesar, Gaul $>$, where the latter is to be understood as $\{\{\{$ Caesar $\}, 1\},\{\{$ Gaul $\}, 2\}\}$. Of course the sequence is to consist of the things themselves, not the names of those things, since it would be nonsense to say that 'Caesar' conquered 'Gaul'.

But now how are we to apply this to electrons or photons? If we could form the needed ordered pairs then the elements of the domain would be absolutely distinguishable, since we would then have a bijection from the particles to the first two ordinals. Indeed if there were such a bijection then these could be taken to be names: as in electron 1 and electron ${ }_{2}$. But if this were the case then the particles would be absolutely discernible, not merely weakly discernible — as per point (3) of the Received View on p. 2 above. The relation of weak discernibility can only be applied to entities that are absolutely discernible — and in fact this will apply also to Black's spheres. Satisfaction is in this case not an easy thing to get. Thus the argument given by Saunders (and also Muller and Saunders (2008), Muller and Seevinck (2009) and Muller (2015)) which Dorato and Morganti rely on is unavailable to them. Particles that are in a singlet state and anticorrelated cannot have this anticorrelation interpreted as a relation between two 'weakly discernible' particles. There is nothing intrinsic in the non-product quantum state that is a 'relation', any more than there is something intrinsic in the state that is a property. ${ }^{13}$ (This is in

\footnotetext{
12 It should be noted that the argument to follow does not depend on the details of the translation of the sequence into set theory. It is the pairing with ordinals that is the crux. Also the details of the satisfaction of open sentences does not change in moving to plural quantification. See Boolos (1985).

13 The idea that there is a property 'behind the veil' of the quantum state is what we know of as a hidden variable theory; it seems that those who are trying to think of relations as lying contained in the quantum state are engaged in an extension of this same project. In QM relations get inferred from the probability statistics of the actions of Hermitian operators acting on spatially separated systems.
} 
line with previous criticisms in Dieks and Versteegh (2008), van Fraassen (2007), van Fraassen and Peschard (2008), and Bigaj (2015) among others.)

We may wonder if we can somehow bypass the formalities of satisfaction, at least in the non-quantal case of Black's spheres. Ladyman's (2007a), Ladyman and Ross (2007b), use of basic unlabelled graphs to represent a symmetric, irreflexive relation may suggest such a way. If we say that there is a sphere that bears an irreflexive symmetric relation to something, then we can immediately infer from this that there must be more than one thing: since no thing can bear an irreflexive relation to itself. How many things may be so related? All we know is that it is two or more; we cannot infer that it is limited to just two. Elsewhere it is argued (Heathcote 2020) that there can be up to four Black spheres - arranged in space in a tetrahedral formation (a Platonic solid) - with the same distance between them all. Five would be impossible. In this case a 4-graph (unlabelled nodes) suggests itself. However, this use of graph theory simply assumes the existence of relations — for which see Dipert (1997) — and it is not clear that it is adding anything by way of logically perspicuous explanatory content to the quantum description, nor that it can be added consistently to that formalism. (This takes us into considerations of structuralism that are best dealt with separately: see Heathcote (2013) for some initial reactions.)

What about Berto's argument, which by-passes weak discernibility and goes straight to a claim of non-identity? As an implication we might summarise it as

$$
\text { Countability } \longrightarrow \text { non-identity. }
$$

We can agree that if we are dealing with individuals contained in a set, that the implication is very plausible. Being a little abstract about naming we could even take the name of any element of a set to be the singleton containing it, given the well-foundedness of the set. Then a set that has a cardinality of 3 has three individuals named by the singletons containing the three elements. Then the claim about non-identity will follow trivially.

But what if the entities are not individuals, and so are not elements of a set? Absent such a relation between cardinality and naming the argument cannot get off the ground. So the assertion of the implication is baseless and question-begging. The main problem, of course, is that if we are concerned with indistinguishable entities that we cannot apply labels to them, for labelling requires a bijection, as

Post-measurement descriptions of what has become a product state cannot be read back into the premeasurement non-product states. Since all we have in QM are states of composites, it is very hard to see where this definite relation might lie. If one wants to base one's metaphysics on relations then there are some high hurdles in QM yet to clear. The issue of entanglement is discussed again in section 4 . 
we saw on p. 2 (based on French and Krause (2006) ch. 5). But Berto does just this.

Of course we might wonder if Berto has not forestalled this response with his claim (made in the first sentence of the quoted passage) that he will resolve this naming issue, invoking the argument of the paper Breckenridge and Magidor (2012). Since that paper contains an audacious view on the use of some names, and since it hasn't hitherto been brought into this debate, it is worth seeing how it might figure in the case in question.

\section{Arbritrary ReFERENCE}

Arbitrary reference is needed, it is claimed, in instantial reasoning involving universal and existential generalisation. The theory that Breckenridge and Magidor offer is that when we say: 'let "Jean" designate an arbitrary person' we may conclude something about all people: say that they are mortal, or have a DNA autosome. In instantial reasoning the name is used to pick out an arbitrary object of the domain, but which object is picked out, we do not know. So we have no idea who 'Jean' is. (It is worth noting at the outset that Breckenridge and Magidor are working within conventional first order logic, so issues of plural quantification do no enter in.)

Note that when we are dealing with instantial reasoning the things to be named are members of a domain and so we already have an assurance, given that they are thus members of a set, that we are dealing with individuals, and therefore that these individuals are able to receive names. In the case of 'Jean' it is assumed that the domain consists of persons, or in the following case, that it contains numbers.

What, for example, does ' $n$ ' refer to following the stipulation that $n$ be an arbitrary number? In this paper, we argue that ' $n$ ' refers to a number - an ordinary, particular number such as 58 or 2,345,043. Which one? We do not and cannot know, because the reference of ' $n$ ' is fixed arbitrarily. (Breckenridge and Magidor (2012) p. 378.)

One might protest that there is a semantic oddity here: if the reference of ' $n$ ' is arbitrary then how can it also be said to be fixed? What is it fixed by? Aren't these contradictory characteristics? And also note that we 'cannot know', thus it is impossible to know, which number has been so referred to, indicating that whatever means there are for fixing a reference on an arbitrary object it can have no features that are able to be consciously or unconsciously deployed by us, no intentionality. Furthermore, note that in the case of ' $n$ ' denoting an arbitrary number, the reference-mechanism, whatever that might be, has an infinite number of natural numbers to choose from. We may begin to wonder if 'reference' is the right name for this relation. (If you aren't impressed with it choosing from a countable set of 
numbers then just switch the example to the real numbers.) Also, with the arbitrary reference idea we can never know whether we have referred to the same thing twice, or whether we haven't. So any statement of the form ' $a=b$ ', with $a$ and $b$ arbitrary names, could never be known to be true or false. These are problems, but, with the exception of the last, not problems I am essentially concerned with here. For the sake of the argument I am prepared to go along with the central cases that Breckenridge and Magidor cover. The issue I want to focus on is whether their account, even if it is able to do what they want it to do in standard cases, is able to apply names to arbitrary indistinguishable particles such as are held to exist in the Received View.

In fact Breckenridge and Magidor $d o$ briefly discuss the issue of indiscernible entities in a closing section of their paper (\$3.2) as Berto notes. ${ }^{14}$ But though they mention elementary particles in a footnote (fn 44) their account is only applied to two cases: Max Black's two iron spheres, and the two imaginary roots of $x^{2}+1=$ 0 . Let us take the second case first. The issues are much easier for them here as these roots are conjugate to one another. So we can arbitrarily name one of the roots $i$ and this will, at the same time, ensure that the other root is $-i-$ because of their conjugate character we must have $i+(-i)=0$. So in this case we can say that we have named the two roots simultaneously, in a single act. So we could call it arbitrary pair-reference. We don't have to worry in this case whether we can name the imaginary roots because we have long done so - all the while acknowledging that which of the roots was said to be positive is entirely arbitrary. Even God couldn't say which one is really the positive root! ${ }^{15}$

Score one, we might say, for Breckenridge and Magidor.

For a less abstract example that makes for a harder case imagine a ball set spinning on a table. There are two points on the ball that are not in motion, which we call the poles. If we want to arbitrarily name one of the poles ' + ' then we can, but we have no reason to name the other pole '-- at the same time, because they are not 'opposites' in the way the conjugate roots were. Of course the other pole must exist if the first one does, but one is not the additive inverse of the other. (This completely changes if the poles are also the poles of a magnet - now, once again, the arbitrary choice of one to be ' + ' forces the other to be '-'.) Note that in this case the table 'breaks the symmetry' of this situation: one pole is touching the table, the other isn't. So in this case when we arbitrarily name one pole it is

\footnotetext{
${ }^{14}$ He says: 'Arbitrary reference so understood, as Breckenridge and Magidor $(2012,398)$ highlight, is especially suitable to explain what goes on when we refer to indiscernible objects.' Berto (2017) p. 18. This rather exaggerates what Breckenridge and Magidor claim.

15 The fact that we can and do name the roots, albeit in an arbitrary fashion, as positive and negative, suggests that Ladyman's idea in Ladyman (2007) of representing then by an unlabelled irreflexive symmetric graph must be open to doubt.
} 
distinguished - though we can't know how it is distinguished - from the other by an extrinsic fact: contact or not with the table. If it were spinning alone in empty space there would be no such symmetry breaking and the case would be more difficult: 'north' and 'south' would then simply be arbitrary names.

Now let's consider the Max Black case of the two iron spheres, from Black (1952).

Suppose we choose the name 'iron-one' to denote an arbitrary sphere. Fine, but how can we denote the other sphere? Remember we don't and can't know which sphere was picked out by our first act of arbitrary reference. So we can't know which is the 'other' sphere, and we cannot refer to it either. A second act of issuing an arbitrary name might be referring again to the sphere we have already named. We would never know whether it was or not. Of course we might agree that on the Breckenridge and Magidor theory there is a sphere that is the 'other' sphere, but much good does it do us. And note that if there were three or four such iron spheres - such as was suggested in the previous section - then even this vestigial use of 'other' will fail — and in fact this provides a better analogy with indistinguishable particles in physics where there is no cap on the number: we aren't just dealing with two (for more on this see Heathcote (2021)). The upshot is that even if we agree that the arbitrary reference to a sphere is a genuine case of reference - and it is a big if - that we still don't have the means to guarantee a reference to the other sphere. Here is their own judgement on this case.

Given AR we can easily explain how we successfully fix the reference of 'A': 'A' arbitrary (sic) refers to one of the spheres. One might worry, though, that this is not a particularly impressive achievement on the part of AR. Why not simply accept that 'A' does not successfully refer in this case? (Breckenridge and Magidor (2012) p. 398)

This can be read as an acknowledgement of defeat in this case. (Incidentally the two spheres case seems to be identical to the case of the two poles of a single sphere spinning in empty space. ${ }^{16}$ )

But Max Black's twin spheres example is not even the most difficult case and it does not properly capture the scope of the problem of indistinguishable particles nor it has to be said, did Black claim that it did (his goal was to show that Leibniz's PII was false).

It was Schrödinger who gave the best analogy for the situation with indistinguishable particles (Schrödinger (1950)), though it is better known through the use of it in a book by Mary Hesse (Hesse (1962)).

\footnotetext{
${ }^{16}$ If we accept this then it blocks some of the responses to Black's case, for example that of Hacking (1975).
} 
Suppose one has 100 dollars in the bank and we attempt to use the arbitrary reference idea to refer to one of these dollars. How will it work? Ordinary reference seems to get no foothold. I can't ask which of the $\$ 100$ is the last that I put in, which is the one that is the accumulated interest. I cannot ask the bank for details of the historical trajectory of one of these dollars and how that trajectory might have intersected with that of another dollar. All of this would make no sense. So it is natural to ask whether the arbitrary reference idea has any applicability in this case. Remember that arbitrary reference was meant to attach - by some means, never specified - to a particular thing in the domain, a particular natural number, person, etc, even though we can't know which one. But the idea of a 'particular dollar' looks very dubious in the current situation. A dollar in the bank is not like a person, a finite set of sets, or like one number in an infinity of numbers. A dollar in the bank lacks individuality: identity conditions don't obtain. If the bank robs me at midnight of my entire $\$ 100$ but repents and replaces it with $\$ 100$ at 12.01 then I have the same now as I lost. There is no difference. It would be absurd to protest 'But where is $m y$ \$100?' Neither self-identity nor individuality exist here, so arbitrary reference doesn't work either.

Of course this example shows in addition, contra Berto's central claim, that one can have a number of indistinguishable entities - that is that they can have a cardinality (there are, after all, 100 dollars) even if no ordinality, for there is no way that we can count them in the ordinal sense while they are simply dollars in a bank. (Of course we can count bank notes, but that represents the dollars by entities that are not indistinguishable.) We can (following Schrödinger) model the idea of bosons by thinking of different accounts, let us say five, at the same bank. Then if we have a thousand dollars there are many ways those thousand dollars can be distributed over the five accounts - while the dollars so distributed are otherwise indistinguishable: they are simply occupation numbers for the accounts/states.

The case of the money in the bank makes for a good analogy with bosons, but is not correct for fermions since it does not incorporate the Pauli exclusion principle. ${ }^{17}$ Schrödinger gave an analogy for fermions in his (1950) that does so. We give this as a final example. Suppose we have a newly-founded chess club that has 10 vacancies, all of the same kind. These vacancies, or better said, positions in the club, can be filled only by a person not already a club member. No one can join twice. If Mary joins the club she fills one of these vacancies, but then she is not eligible to fill another vacancy - and this is the crux of the matter, all of the positions are indistinguishable. It makes no sense to think of vacancies being rearranged, or of Mary as having filled one vacancy as opposed to some other, or

\footnotetext{
17 Hesse claimed that it did in Hesse's (1962), and this was a mistake. Unfortunately many have followed her lead. But Schrödinger had gotten the matter right in his (1950).
} 
of her moving from one vacancy to another: if she's in, she's in. It is because of this that we have an analogy with the Pauli exclusion principle, in the form that Pauli formulated in 1925, in that Mary is only able to join the club once. Only one vacancy can be filled by any person. ${ }^{18}$. Note that in this analogy to fermions it is the positions that correspond to the particles; the people represent the states. As already noted: the particles are indistinguishable and so are the club positions: it makes no sense to think of vacancies as being swapped, interchanged or permuted; it makes no sense to count the vacancies by ordinals, as in the 4th and the 9th and yet they have a cardinality: there are 10 vacancies. Here it is in Schrödinger's own words:

The example may seem odd and inverted. One might think, "Why cannot the people be the electrons and various clubs [this should be 'vacancies in clubs'] their states? That would be so much more natural." The physicist regrets, but he cannot oblige. And this is just the salient point: the actual statistical behaviour of electrons cannot be illustrated by any simile that represents them by identifiable things. That is why it follows from their actual statistical behaviour that they are not identifiable things. Schrödinger (1950) p. 207

A logic of such vacancies would be a logic of particles. ${ }^{19}$ Are the vacancies able to be arbitrarily named, as per the theory of Breckenridge and Magidor? No, the positions are indistinguishable and so can't be named at all, let alone arbitrarily named. The Breckenridge and Magidor position may have some plausibility in the case of individuals in a set-theoretic domain but it can get no purchase on indistinguishable particles. As Schrödinger said in 1961: 'The fact that the individual particle is not a well-defined permanent object of determinable identity or individuality is probably admitted by most theoreticians just as they admit the reasons described here for the complete inadequacy of the representation.' (1961) p. 62.

If, after this digression, we return to Berto's argument cited on p. 7 - with its reliance on the arbitrary names idea of Breckenridge and Magidor - we can see that it breaks down at the first step: the naming of an indistinguishable entity. The claim made by Berto (and also Dorato and Morganti) that there can't be entities that are indistinguishable which yet have a cardinality has been shown to be false.

Thus far we have dealt only in analogies to indistinguishable particles in QM - but this only takes us so far. In the next section we confront the problem by giving a direct argument.

\footnotetext{
18 It would not be difficult to adapt this to the antisymmetric subspace idea of Dirac and Weyl.

19 Can a single vacancy be a vacancy for two clubs - say a maths club, that has to have one member in the chess club? In other words an intersection of members and vacancies? I see no reason why not.
} 


\section{THE ARGUMENT FOR INDISTINGUISHABILITY}

In Weyl (1931) we find the following paragraph: (I've given a more technical discussion of this paragraph and how it fits into Weyl's larger discussion in Heathcote (2020). But the main point can be made quite simply and it is worth doing so.)

The only characteristic numbers associated with the system space $\left\{\Re^{2}\right\}$ are those of the type $E_{1}+E_{2}$, each of which appears exactly once, but the sub-space $\left[\Re^{2}\right]$ has simple characteristic numbers of the type $2 E_{1}$ in addition to these. Hence if Nature decides in favour of $\left\{\Re^{2}\right\}$ both individuals can never be simultaneously in the same quantum state with energy $E_{1}$ - assuming this energy level for the individual system is non-degenerate. That $E_{1}+E_{2}$ occurs only once in $\left\{\Re^{2}\right\}$ and only once in $\left[\Re^{2}\right]$ means : the possibility that one of the identical twins Mike and Ike is in the quantum state $E_{1}$ and the other in the quantum state $E_{2}$ does not include two differentiable cases which are permuted on permuting Mike and Ike; it is impossible for either of these individuals to retain his identity so that one of them will always be able to say "I'm Mike" and the other "I'm Ike". Even in principle one cannot demand an alibi of an electron! In this way the Leibnizian principle of coincidentia indiscernibilium holds in quantum mechanics. (Weyl (1931) p. 241.)

This requires some explanation. The full Hilbert space for two particles is denoted as $\mathfrak{R}^{2}$. But as noted at the beginning of this paper we are only interested in two sub-spaces, the symmetric, for bosons, denoted here as $\left[\Re^{2}\right]$, and the antisymmetric, for fermions, denoted $\left\{\Re^{2}\right\}$. Weyl has simplified the discussion to the eigenvalues of only one operator, the energy. (The phrase 'characteristic numbers' in the above passage is due to the translator: it means eigenvalues.) The crucial point is stated in the opening sentence: the only energy eigenvalues for a pair of electrons in a Helium atom would be of the kind $E_{1}+E_{2}$. If we were looking at the eigenvalues for a pair of bosons, however, we would have the possibility of them both being in the same eigenstate with eigenvalues given as $2 E_{1}$, or $2 E_{2}$. But for electrons this is not possible. Thus there are more eigenvalues available to bosons, more possibilities. For fermions in $\left\{\mathfrak{R}^{2}\right\}$ there is here only one possibility: the eigenvalue sum $E_{1}+E_{2}$, and it 'appears exactly once'. So there is only one way of realising this eigenvalue: one electron must be in $E_{1}$ and the other in $E_{2}$. This represents the only possibility. There are not two copies of the eigenvalue sum, as there was in the unreduced space $\mathfrak{R}^{2}$ : one configuration with the individuals in one set of eigenstates and another with them permuted. There are not two copies of the eigenvalue sum $E_{1}+E_{2}$ that electrons could be permuted in, and thus they 
are indistinguishable particles: an electron is in $E_{1}$ another is in $E_{2}$. The same argument applies to bosons in $\left[\Re^{2}\right]$, since $E_{1}+E_{2}$ appears in $\left[\Re^{2}\right]$ just once, they also are indistinguishable. Two possibilities have been collapsed to one, meaning that the particles are not distinguishable. This is what Weyl means in saying that 'In this way the Leibnizian principle of coincidentia indiscernibilium holds in quantum mechanics.' It holds not of the electrons themselves, but of the available eigenstates and eigenvalues, the possibilities: two have been collapsed to one.

When French and Krause quoted this paragraph they truncated it severely so that it began mid-sentence and didn't include the final sentence. It was quoted as:

... the possibility that one of the identical twins Mike and Ike is in the quantum state $E_{1}$ and the other in the quantum state $E_{2}$ does not include two differentiable cases which are permuted on permuting Mike and Ike; it is impossible for either of these individuals to retain his identity so that one of them will always be able to say "I'm Mike" and the other "I'm Ike". Even in principle one cannot demand an alibi of an electron! (French and Krause (2006) p. 105) ${ }^{20}$

This misses out the crucial point that the eigenvalue sum $E_{1}+E_{2}$ only appears once - and it gives the impression that the central argument is something to do with permuting Ike and Mike - rather than the impossibility of doing this when they are electrons! ${ }^{21}$ It also creates a mystery as to why Weyl says that the Leibniz principle is maintained - a point that appears to have created a second level of confusion (see Heathcote (2020)). French and Krause focus their attention on what Weyl says later in Weyl (1949), which, though important, does not give an argument for indistinguishability. Thus French and Krause (2006) contains no coherent argument for indistinguishability.

The reduction to the antisymmetric space implies the Pauli exclusion principle (the converse implication does not hold). If we confine ourselves just to pure states then the states of any system, fermion or boson, are distinguishable: states are distinguishable even though particles are not (as Schrödinger and Weyl emphasised).

It is worth noting also, since our subject here is identity, that the antisymmetry that determines the fermion space is itself an identity:

$$
\psi(i j)=-\psi(j i) .
$$

\footnotetext{
${ }^{20}$ Berto (2017) p. 4 gives the same truncated quote but introduces five punctuation errors into the quotation, which further serve to distort the meaning.

${ }^{21}$ In the German the names were Hans and Karl. This was changed to Mike and Ike by the translator after a popular cartoon by Rube Goldberg 'Mike and Ike (They look alike)'. In 1927 this cartoon was turned into a number of feature films starting with Dancing Fools — ironically starring two actors who did not look alike! This change ensured that the idea would be conveyed to audiences in 1930's America.
} 
Thus there is no immediate prospect of getting rid of identity relations.

\section{ORDINARY OBJECTS}

Can we now go further? What about ordinary middle-sized objects: tables, chairs, people, galaxies? Do they have self-identity? The first thing to say is that one moral from a century of discussions on identity, Theseus' ship, disagreements about constitution as identity, etc, is that such objects appear to have no precise identity conditions. We don't really know where person $A$ ends and the environment for $A$ begins; we don't know where or when $A$ turns into not- $A$. Is the air inhaled and exhaled a part of $A$ ? What about the many trillions of neutrinos that pass through the body every second? What about the cells that die and are passed out of the body? Which of those, if any, are part of $A$ ? Similar problems exist for inanimate objects.

Quantum mechanics tells us that objects, or the small parts of objects, will be entangled with their environments with which they have interacted. What is the object and what is the environment? Is there a meaningful difference? The identity of indiscernibles will not really help here, since we first have to answer the question as to what are $A$ 's properties, which is precisely the problem. Suppose we bypass all of these questions and simply agree for the sake of argument that identity for ordinary middle-sized objects — the objects that we call classical — is not determinate. In fact, Breckenridge and Magidor attempt to apply their theory to objects that are indeterminate, namely those that are vague. So can their view be made to work here? According to them arbitrary reference resolves the vagueness of whether something is tall in an arbitrary way by simply picking an arbitrary height and labelling it as 'tall'. As they say :

Consider the predicate 'tall' and the range of admissible precisfications for it. The proposal is that 'tall' had its reference fixed arbitrarily to one of these properties. This means, that 'tall' refers to a particular sharp property (so it does not make for any failures of classical logic). But the fact that we do not and cannot know which property 'tall' denotes has nothing in particular to do with vagueness: it has to do with the ignorance associated with applications of AR more generally ... (Breckenridge and Magidor (2012) p. 399)

Of course this suggestion faces problems. For example the height arbitrarily picked out may be a height that no one would regard as tall, such as the Planck length. But since we can't know what height has been picked we may let this pass. Suppose that we attempt to apply this solution to the problem of the vagueness of middlesized objects: where do I end and where does not-me begin? Suppose that that 
is arbitrarily fixed in the way we've already seen. We can't know what has been precisely fixed as me, either spatially or temporally, but, apart from this, does it give us all we need? Well suppose that arbitrary reference is used a second time, by someone else, or by the 'same' person (and we quickly pass over the problem that this induces) at another time or place, so as to form a sentence of the kind ' $a=b$ ' : is such a sentence true or false? Well we can't actually know, because we can't know what precisifications have been induced by either of these invocations of arbitrary reference. But if we reckon the possible precisifications to have the cardinality of the continuum then the probability of the precisification associated with ' $a$ ' and the precisification associated with ' $b$ ' coinciding would be 0 - because the set in the event space has measure 0. So statements of the form ' $a=b$ ' will have a probability of 0 of being true. One precisification of what is me will have zero probability of coinciding with another precisification of what is me. This applies both synchronically and diachronically — and it applies also to any other middle-sized objects.

But before we move on it is worth looking at the application of the Arbitrary Reference idea to the Geach-Unger Problem of the Many (Unger's term, from Unger (1980)). ${ }^{22}$

The argument was put succinctly in Geach (1980) and at length in Unger (1979) and (1980). The argument, in Geach's version, concerns a cat, Tibbles, and 1,000 of his hairs. Let $c$ be the name for the 'largest continuous mass of feline tissue on the mat', and let $h_{n}$ be the name for the $n$-th hair of the 1000 . Let $c_{n}$ be the proper part of $c$, namely $c-h_{n}$; repeat for each of the 1,000 hairs. He reasons:

Moreover, fuzzy as the concept cat may be, it is clear that not only is $c$ a cat, but also any part $c_{n}$ is a cat: $c_{n}$ would clearly be a cat were the hair $h_{n}$ to be plucked out, and we cannot reasonably suppose that plucking out a hair generates a cat, so $c_{n}$ must already have been a cat. So, contrary to our story, there was not just one cat called "Tibbles" sitting on the mat; there were at least 1,001 sitting there! Geach (1980) p. 215

Of course Geach takes that conclusion to be absurd. So what to say instead? The right thing to say, he says, is that there is one cat on the mat, but many continuous lumps of feline tissue. "Tibbles" is a name for the cat. He says: "Everything falls into place if we realize that the number of cats on the mat is the number of different cats on the mat; and $c_{13}$ and $c_{279}$ and $c$ are not three different cats, they are one and the same cat. Though none of these 1,001 lumps of feline tissue is the same lump of feline tissue as another, each is the same cat as any other; . . '

\footnotetext{
22 This application was not considered in Breckenridge and Magidor (2012). My thanks to a reader for the journal for suggesting the inclusion of this work.
} 
Unger has a very different response to this kind of problem. For him the conclusion must be that there is either no cat on the mat (nor any mat), or that there are a myriad of cats on a myriad of mats. He inclines to the first of these solutions, which he calls Parmenidean. If one inclines to the standard view that neither of these are true and that there is only one cat he suggests that one has to come up with a selection principle.

A selection principle, one that is both applicable and correct, will single out, or select, from among the entities in a situation, those that satisfy a certain description or possess a certain property (or, in particular, that are of a certain kind). Unger (1980) p. 449.

Unger takes such a selection principle as needing to offer a reason for favouring one particular candidate (for being the cat, for example). When no such reason presents itself he declares that there cannot be one thing present: one cat, one stone, or one cloud. But Breckenridge and Magidor could offer a different kind of solution: the selection is arbitrary. Arbitrary reference picks out one lump of continuous feline flesh as Tibbles — or, rather, picks out one particular such lump by using the name 'Tibbles'. Someone else may pick out another such lump, plus or minus this or that hair. In neither case can we know what precisely is picked out. But both will agree that there is only one cat on the mat. This looks like a distinct victory for arbitrary reference. ${ }^{23}$

But note what it does not give us. It does not, as previously noted, give us the identities we require for individuality. If I use arbitrary reference at some time $t_{1}$ to give a referent to the name 'Tibbles', then as we saw, a second use at time $t_{2}$ will have probability 0 of being the same precisification. And so we will have: Tibbles 1 $\neq$ Tibbles$_{2}$. And we will have the same lack of identity if the first precisification is done by person $A$ at a given time $t_{1}$ and a second person $B$ also at $t_{1}$.

To this it may be objected that we are not interested in a Tibbles subscripted but simply Tibbles. Surely, it may be said that Tibbles = Tibbles? Surely that is clear? The problem though is that it is not clear; it is ambiguous depending on what the precisifications are, since they determine the reference.

What if we stipulate that it is the same precisification? The problem then becomes the identity conditions for precisifications. Geach does not provide much of a guide here but Unger is much more forthcoming and, though Unger avoids mathematical terminology, the mathematical idea is clear: for a normal object, such as

${ }^{23}$ Unger thinks that the myriad precisifications may lead us to conclude that there are a myriad of cats but overlooks the fact that no given precisification would have it that there are even two cats on the mat, let alone many. What is plural (as noted by Geach) are the possible precisifications, not the cats. The myriad of possible precisifications creates a disjunction of possibilities that can qualify as the cat, not a conjunctive sum of many cats. 
a cat (or cloud or stone), a precisification is a two dimensional compact surface, an envelope, that has the 'object', and nothing else, as its interior. We might call it a precisification envelope. ${ }^{24}$

The problem is how to make this mesh with quantum mechanics. Can we have a compact surface that disconnects the space into an interior and an exterior that is consistent with what we have seen so far of quantum mechanics?

One problem is the issue of localizability, which arises from the nature of even a single wave function, its support on the space (the part of the space on which the function is non-zero), and is connected with the Heisenberg uncertainty relation. This support can be non-compact (see Hughes (1989) ch. IX for a summary).

But I want to focus on two problem that arise from matters closer to the subject of this paper: particle indistinguishability and inseparability, or entanglement. The definition of the latter in its usual formulation is that a mixed state of a composite quantum system is entangled, or inseparable, if it cannot be written as a convex combination of product states. (See Bengtsson and Życzkowski (2017) for a modern account.)

Schrödinger highlighted the entanglement problem with his cat paradox, in which the cat, the box, and the measurement device become entangled.

As soon as the systems begin to influence each other, the combined function ceases to be a product and moreover does not again divide up, after they have again become separated, into factors that can be assigned individually to the systems. Thus one disposes provisionally (until the entanglement is resolved by an actual observation) of only a common description of the two in that space of higher dimension. This is the reason that knowledge of the individual systems can decline to the scantiest, even to zero, while knowledge of the combined system remains continually maximal. Best possible knowledge of a whole does not include best possible knowledge of its parts - and that is what keeps coming back to haunt us. (Schrödinger (1935) p. 167)

To put this in the terms of the Geach-Unger precisification: any precisification envelope that contains the cat must also, in virtue of their inseparability, contain the apparatus as well. We can then further ask, as Schrödinger doesn't in this passage, whether the apparatus is also connected to other elements in the world, and thus the cat also. The process can be iterated.

Much of the discussion of this issue in the past sixty years has been conducted with only two entangled particles (i.e. bipartite systems) where the entanglement

\footnotetext{
24 'Compactness' is a topological term roughly meaning a bounded, closed space. The surface of a 2-sphere is a good example.
} 
is limited by the Tsirel'son bound. But the current evidence is that as the number of particles increases (i.e. multipartite systems) the nature of entanglement becomes much more complex and is able to exceed such a bound (Pérez-García et al (2008)). ${ }^{25}$ Moreover, the nature of the entanglement itself can change as the dimensions of the tensor (Hilbert) space rises. The phenomenon in higher dimensions is called 'bound entanglement' (bound as opposed to free, the familiar form): in this the entangled state cannot be 'distilled' to a singlet state - i.e. reducing the 'noise' in the state, using local operations and classical communication (LOCC), does not turn it into the familiar EPR state. ${ }^{26}$ An author of the idea says:

However, when we considered more complicated systems [than bipartite systems], we obtained surprising results. Paradoxically, nature subject to a purely mathematical treatment revealed the physical peculiarity of entanglement: namely, that the environment may "pollute" the pure entanglement in such a way that it will not be possible to cleanse it with the help of LOCC [local operations and classical communications]. ${ }^{27}$ (Horodecki (2010) p. 23)

This implies that we can have mixed states (pure states subject to a noisy environment) that are entangled but are such that the effect of the noise cannot be reduced by local means. If a system contains only two qubits then it can be distilled, but there are 4-qubit states that cannot be. There are 4-qubit systems where the components are far from one another such that the entanglement cannot be distilled in any pair. Thus for more complex systems the effect of the environment cannot be removed. (For the additional complexity that takes place with just three particles see Bengtsson and Życzkowski 2017, ch. 17.)

Put in the language of Geach-Unger: there is no precisification envelope that can go around any component that does not go around all.

We end on the matter that has taken up most of this paper: the indistinguishability of particles. We have already spent some time on arguments and analogies here so it is possible to make the point briefly. Suppose we have an electron that is part of an atom in a body - by which we mean that there is a state of this complex system that is occupied by an electron. We cannot distinguish this electron from an electron that occupies a state that is not within the body. It is meaningful to speak

\footnotetext{
25 Tsirel'son's bound is equivalent to Grothendieck's constant used in the paper. The complexity of multipartite entanglement is well discussed in the review Horodecki et al (2009). This discovery was initiated by Peres (1996).

26 This notion was introduced in Horodecki et al (1998). See also and Smolin (2001). Bounded entanglement was confirmed in the laboratory in 2010, see Barreiro et al (2010).

27 They continue: 'This result astonished the physicists. It transpired that the structure of entanglement is not uniform!'
} 
of there being two electrons, but not meaningful to speak of them as having an identity, of one as opposed to the other. (To repeat a point already made: the (pure) states may be spoken of as distinguishable, but not the occupants of the states.) But then it is meaningless to speak of particular electrons as being part of the body. Thus there is no precisification envelope that can go around a body that has one electron on the inside and the other on the outside. If we could do it then we could tighten the envelope and distinguish the electrons as finely as one likes - but we already know that this is impossible.

All of this makes the issue of separating any macroscopic object, such as a person, from the environment an impossible task. Neither states nor particles can be regarded as determining a body as lying entirely within a precisification envelope. Therefore they cannot be individuated. ${ }^{28}$ This failure is a failure of synchronic identity conditions. But if synchronic identity can't be made determinate, then it can't be determinate across time either, since if an entity is indeterminate at $t_{1}$ then whatever it is at $t_{2}$ cannot be determined to be the same as it was at $t_{1}$. (This point was made earlier but it bears repeating.) Thus diachronic identity is indeterminate as well.

The moral is that individuality is a Procrustean bed for middle-sized objects. They simply can't fit it, synchronically or diachronically.

It may seem as though we are on the way to embracing the notion that selfidentity is vague - a view argued against by Evans in his (1978). But this is not the conclusion that I want to draw. I want to suggest that these middle-sized objects simply do not have identity conditions, vague or otherwise, and therefore no self-identity. They are not individuals. ${ }^{29}$ This was the view of F. H. Bradley who said: 'It is hard to say what, as a matter of fact, is generally understood when we use the word "thing." But, whatever that might be, it seems now undermined and ruined. . . . For a thing to exist it must possess identity ; and identity seems a possession with a character at best doubtful.' (Bradley (1916) ch. VIII)

This does not mean that there are no individuals of any kind - and Bradley we can be sure was only concerned with ordinary middle-sized objects. Whether there are or not has to be considered for each class of objects. So we may ask: what might self-identity apply to? Numbers would be a good candidate for things that are individuals, as would pure sets, anything that can be precisely specified. But a discussion of this is beyond the scope of this paper. ${ }^{30}$

\footnotetext{
${ }^{28}$ For the interaction between entanglement and indistinguishability see Wiseman and Vaccaro (2003) or Tóth and Gühne (2009). This is an area of ongoing research.

29 There is a persuasive refutation of Evans argument in Lowe (1998). The view I am suggesting is one of the possible counterarguments that Lowe considers.

${ }^{30}$ People are not suitable to be members of sets. As Groucho Marx (almost) said: You wouldn't want to be a member of any set that would have you as a member.
} 
The conclusion we have reached is of a physical world of non-individuals, both at the micro- and macro- level. Weyl touched on this idea; 'Hence these real symmetry operators represent physical quantities of the physical system of $f$ equivalent individuals, whose total system space is $\mathfrak{R}^{f}$; quantities of this kind are unknown in classical physics and cannot be pictured in terms of the usual spatial and temporal models.' (Weyl (1931) p. $287^{31}$ )

\section{ConClusion}

If $x$ has self-identity, so that $x=x$ is meaningful and true, then $x$ has a property that no other entity has, namely that of being identical with $x$. So it is individuated by that property. Thus in a set that includes $x$ there will be the singleton that contains just $x$. The singletons are bijectively mapped to their members and so, in an abstract sense can count as names of those things, thus $x$ is nameable. But we have presented an argument from Schrödinger that there are entities that are not individuals (e.g. amounts of money in the bank, positions in a team, quantum particles) and that therefore do not have self-identity (by contraposition on the above) and are unnameable. Nevertheless these things can have a cardinality - we can have 100 dollars in the bank, there can be three vacant positions, two electrons are in a Helium atom. This is sufficient to show that the mere fact of having a cardinality does not imply self-identity or individuality. Therefore Berto's argument is refuted. Berto's argument relied on a naming strategy, described in Breckenridge and Magidor (2012), and so we were led to consider this theory. We found that it could not serve the purpose that Berto intends. In fact, as we can see from the above, nameability implies individuality. Thus Berto's argument was circular.

That quantum particles are entities lacking self-identity, individuality and nameability is called the Received View of Quantum Mechanics. We have extended the Received View to the macroscopic objects that they make up. Particles are indistinguishable within their kinds, as physicists have maintained for nearly 100 years. They are not individuals: but in this they take middle-sized objects down with them.

\footnotetext{
31 In a footnote to this Weyl cites Dirac's (1929) paper on many electron systems.
} 
Acknowledgments: My thanks to the two readers of this journal who made some excellent suggestions for improvements.

\section{Declarations:}

Funding: Not applicable

Conflicts of Interest: None

Availability of Data: Not applicable

Code availability: Not applicable

Author's Contributions: Not applicable

Research involving human participants and/or animals: Not applicable

Informed consent: not applicable. 


\section{REFERENCES}

Barreiro, J. T., Schindler, P., Gühne, O., Monz, T., Chwalla, M., Roos, C. F., ... Blatt, R. (2010). Experimental multiparticle entanglement dynamics induced by decoherence. Nature Physics, 6, 12, 943-946. doi:10.1038/nphys1781

Bengtsson, I. and Życzkowski, K. (2017). Geometry of Quantum States, second edition, Cambridge: Cambridge University Press. doi: 10.1017/9781139207010

Berto, F. (2017). Counting the Particles: Entity and Identity in the Philosophy of Physics. Metaphysica, 18, 1, 1-21.

Black, M. (1952), The Identity of Indiscernibles, Mind 61: 153-164.

Blizzard, W. D. (1989). Multiset Theory. The Notre Dame Journal of Formal Logic, 30, 1, 36-66.

Boolos, G. S. (1984). To Be Is To Be a Value of a Variable (or to Be Some Values of Some Variables), The Journal of Philosophy, 81(8): 430-50; (reprinted in Logic, Logic, Logic. ed. Richard Jeffrey. Cambridge, Mass.: Harvard University Press) doi: 10.2307/2026308

Boolos, G. S. (1985a). Nominalist Platonism, Philosophical Review, 94. 3: 327344; reprinted in Boolos 1998. doi: 10.2307/2185003

Boolos, G. S. (1985b). Reading the Begriffsschrift, Mind, 94, 331-344, reprinted in Boolos 1998.

Bradley, F. H. (1916). Appearance and Reality: a metaphysical essay, (sixth impression) London: George Allen and Unwin.

Breckenridge, W. and Magidor, O. (2012). Arbitrary Reference, Philosophical Studies, 158, 377-400.

Bueno, O. (2014). Why Identity Is Fundamental. American Philosophical Quarterly, $51: 325-332$.

Cartwright, R. L. (1994). Speaking of Everything, Noûs, 28, 1, 1-20

Cartwright, R. L. (2001). A Question about Sets, in Alex Byrne, Robert Stalnaker, and Ralph Wedgwood (eds.), Fact and Value: Essays on Ethics and Metaphysics for Judith Jarvis Thomson, Cambridge, MA: MIT Press, pp. 29-46

Dalla Chiara, M. L. and Toralda Di Francia, G. (1995). Identity Questions from Quantum Theory. In: Gavroglu K., Stachel J., Wartofsky M.W. (eds) Physics, Philosophy, and the Scientific Community. Boston Studies in the Philosophy of Science, vol 163. Springer, Dordrecht 
Dalla Chiara, M. L. (1985). Some Foundational Problems in Mathematics suggested by Physics, Synthese, 62, 303-315.

Dieks, D. and Versteegh, M. A. M. (2008). Identical Quantum particles and Weak Discernibility. Foundations of Physics, 38, 923-34.

Dieks, D. (2020). Identical Quantum Particles, Entanglement, and Individuality, Entropy, 22, 134, 1-16. doi: 10.3390/e22020134

Dipert, Randall R. (1997). The Mathematical Structure of the World: The World as Graph. The Journal of Philosophy, 94, 7, 329-58.

Dirac, P. A. M. (1926). On the Theory of Quantum Mechanics, Proceedings of the Royal Society of London A. 112, 661-77.

(1929). Quantum Mechanics of Many-Particle Systems. Proceedings of the Royal Society of London A. 123, 714-33.

Dorato, M. and Morganti, M. (2013). Grades of Individuality. A pluralistic view of identity in quantum mechanics and the sciences. Philosophical Studies, $163,591-610$.

Evans, G. (1978). Can There Be Vague Objects?. Analysis, 38, 4, 208.

Fraassen, Bas C. and Peschard, Isabelle (2008). Identity over time: Objectively, subjectively. Philosophical Quarterly 58 (230): 15-35.

French, S. and Krause, D. (2006). Identity in Physics: a Historical, Philosophical and Formal Analysis. Oxford: Clarendon Press.

French, S. and Krause, D. (2010). Remarks on the Theory of Quasi-Sets. Studia Logica, 95: 101-124. doi: 10.1007/s11225-010-9249-3

Geach, P. T. (1980). Reference and Generality, third edition, Ithaca: Cornell University Press.

Hacking, I. (1975). The Identity of Indiscernibles. The Journal of Philosophy, 72, 9, 249. doi:10.2307/2024896

Hallett, M. (1984). Cantorian Set Theory and Limitation of Size. Oxford: Oxford University Press.

Heathcote, A. (2014) On the Exhaustion of Mathematical Entities by Structures, Axiomathes. June, 24, 2, pp 167-180. doi: 10.1007/s10516-013-9223-6

Heathcote, A. (2020). Multiplicity and Indiscernibility, Synthese on-line first. doi: $10.1007 / \mathrm{s} 11229-020-02600-8$ 
Heathcote, A. (2021). Five Indistinguishable Spheres, Axiomathes, Online-first, doi: 10.1007/s10516-020-09531-6

Hesse, M. (1963). Models and Analogies in Science, London: Sheed and Ward; reprinted 1966 Notre Dame, University of Notre Dame Press.

Horodecki, M., Horodecki, P., and Horodecki, R. (1996). Separability of mixed states: necessary and sufficient conditions. Physics Letters A, 223, 1-2), 1-8. doi: 10.1016/s0375-9601(96)00706-2

Horodecki, M., Horodecki, P., Horodecki, R. (1998). Mixed-State Entanglement and Distillation: Is there a Bound Entanglement in Nature?. Physical Review Letters. 80, 24, : 5239-5242. doi:10.1103/PhysRevLett.80.5239

Horodecki, R., Horodecki, P., Horodecki, M. and Horodecki, K. (2009). Quantum Entanglement. Reviews of Modern Physics, 81, 2, 865-942. doi: 10.1103/revmodphys.81.865.

Horodecki, R. (2010). Bound entanglement - mysterious invention of nature. Europhysics News, 41, 6, 21-24. doi: 10.1051/epn/2010603

Hughes, R. I. G. (1989). The Structure and Interpretation of Quantum Mechanics. Camb: Mass: Harvard University Press.

Jantzen, B. C. (2011). No Two Entities Without Identity, Synthese, 181, 3, 433-50. doi: 10.1007/s11229-010-9717-3.

Jantzen, B. C. (2017). Entities without Identity: a Semantical Dilemma, Erkenntnis, online first. doi: 10.1007/s10670-017-9958-3

Krause, D. and Arenhart, J. R. B. (2018a). Quantum Non-Individuality: Background Concepts and Possibilities. in The Map and the Territory: exploring the foundations of science, thought and reality, ed. Springer doi: 10.1007/9783-319-72478-2

Krause, D. and Arenhart, J. R. B. (2018b). Is identity Really so Fundamental? Foundations of Science. published on-line July 2018.

Ladyman, J. (2007a). On the Identity and Diversity of Objects in a Structure. Aristotelian Society Supplementary Volume, 81, 1, 23-43.

Ladyman, J. and Ross, D. (2007b). Every Thing Must Go: Metaphysics Naturalised, Oxford: Oxford University Press.

Linnebo, Øystein and Agustín Rayo, (2012). Hierarchies Ontological and Ideological, Mind, 121(482): 269-308. doi:10.1093/mind/fzs050 
Lowe, E. J. (1998). The Possibility of Metaphysics: Substance, Identity and Time. Oxford: Clarendon Press.

McGinn, C. (2000). Logical Properties: Identity, Existence, Predication, Necessity, Truth. Oxford: Clarendon Press.

Muller, F. A. (2015). The Rise of Relationals, Mind, 124: 201-237.

Muller, F. and Saunders, S. (2008). Discerning Fermions, British Journal for the Philosophy of Science, 59: 499-548.

Muller, F. A. and Seevinck, M. P. (2009). Discerning elementary particles. Philosophy of Science, 76, 179-200.

Parker-Rhodes, A. F. (1981). The Theory of Indistinguishables, Synthese Library, Dordrecht Holland, Reidel.

Peres, A. (1996). Separability Criterion for Density Matrices. Physical Review Letters, 77, 8, 1413-1415. doi: 10.1103/physrevlett.77.141

Pérez-García, D., Wolf, M. M., Palazuelos, C., Villanueva, I., and Junge, M. (2008). Unbounded Violation of Tripartite Bell Inequalities. Communications in Mathematical Physics, 279, 2, 455-486. doi:10.1007/s00220-0080418-4

Quine, W. V. (1986). Philosophy of Logic (second edition). Harvard University Press

Rayo, A..and Uzquiano, G. (2006). Absolute Generality, Oxford: Clarendon Press.

Savellos, E. E. (1990). On Defining Identity, Notre Dame Journal of Formal Logic, $31,3,476-84$.

Schrödinger, E. (1935). The Current Situation in Quantum Mechanics (translation reprinted in Quantum Theory and Measurement, ed. John Wheeler and W. Zurek. Cambridge: Cambridge University Press.)

(1950). What is an elementary particle?, Endeavour, 9, 109-116. (Reprinted in Castellani, Elena (ed) Interpreting Bodies: Classical and Quantum Objects in Modern Physics, Princeton: Princeton University Press, 1998.)

(1961). Our Image of Matter, in On Modern Physics, 45-66, New York: Clarkson N. Potter. 1961.

Schumener, Erica (2017). The Metaphysics of Identity: Is Identity Fundamental? Philosophy Compass 2017; 12: e12397. doi: 10.1111/phc3.12397 
Smolin, J. A. (2001). Four-party unlockable bound entangled state. Physical Review A. 63, 032306.

Toraldo di Francia, G. (1978). What Is a Physical Object? Scientia 113, 57-65.

Tóth, G., and Gühne, O. (2009). Entanglement and Permutational Symmetry. Physical Review Letters, 102, 17. doi: 10.1103/physrevlett.102.17

Trueman, R. (2014). Eliminating Identity: A Reply to Wehmeier, Australasian Journal of Philosophy, 92, 1.

Unger, P. (1979). Why There Are No People. Midwest Studies in Philosophy, 4, 1, 177-222. doi:10.1111/j.1475-4975.1979.tb00377.x

Unger, P. (1980). The Problem of the Many. Midwest Studies In Philosophy, 5, 1, 411-468. doi: 10.1111/j.1475-4975.1980.tb00416.x

Van Fraassen, B. C. (1991). Quantum Mechanics: an Empiricist View. Oxford: Clarendon Press.

Van Fraassen, B. C. (2007). Structuralism(s) about Science: Some Common Problems. Aristotelian Society Supplementary Volume, 81, 1, 45-61. doi: 10.1111/j.14678349.2007.00150.x

Wehmeier, K. F. (2012). How to Live without identity — and Why. Australasian Journal of Philosophy, 90, 4, 761-777.

Wehmeier, K. F. (2014). Still Living without Identity: a Reply to Trueman. Australasian Journal of Philosophy. 92, 1, 173-5.

Weyl, H. (1931). The Theory of Groups and Quantum Mechanics (trans. of Gruppentheorie und Quantenmechanik, second edition, by H. P. Robertson). London: Methuen (reprinted 1950 unaltered by Dover, N.Y.)

(1949). Philosophy of Mathematics and Natural Science. Princeton, N. J.: Princeton University Press.

Williamson, T. (2003). Everything. Philosophical Perspectives, 17: 415-465. doi: 10.1111/j.1520-8583.2003.00017.x

Wiseman, H. M., and Vaccaro, J. A. (2003). Entanglement of Indistinguishable Particles Shared between Two Parties. Physical Review Letters, 91, 9. doi: 10.1103/physrevlett.91.097902 